\title{
Erratum
}

Stuart G. Baker* and Karen S. Lindeman

\section{Erratum to Revisiting a Discrepant Result: A Propensity Score Analysis, the Paired Availability Design for Historical Controls, and a Meta-Analysis of Randomized Trials [J Causal Inference DOI: 10.1515/jci-2013-0005]}

*Corresponding author: Stuart G. Baker, National Cancer Institute, Bethesda, MD, USA, E-mail: sb16i@nih.gov Karen S. Lindeman, Johns Hopkins Medical Institutions, Bethesda, MD, USA, E-mail: klindema@jhmi.edu

Erratum in: Journal of Causal Inference 2013;1(1):51-82, DOI: 10.1515/jci-2013-0005

We thank Professor Marcel Zwahlen, University of Bern, Switzerland, for pointing out the following corrections: In Figure 1, the labels for the lines, $T=0$ and $T=1$, should be reversed. Equation (13) should be $\operatorname{pr}(T=1 \mid Z=0)=\operatorname{pr}(R=i)+\operatorname{pr}(R=a)$. 